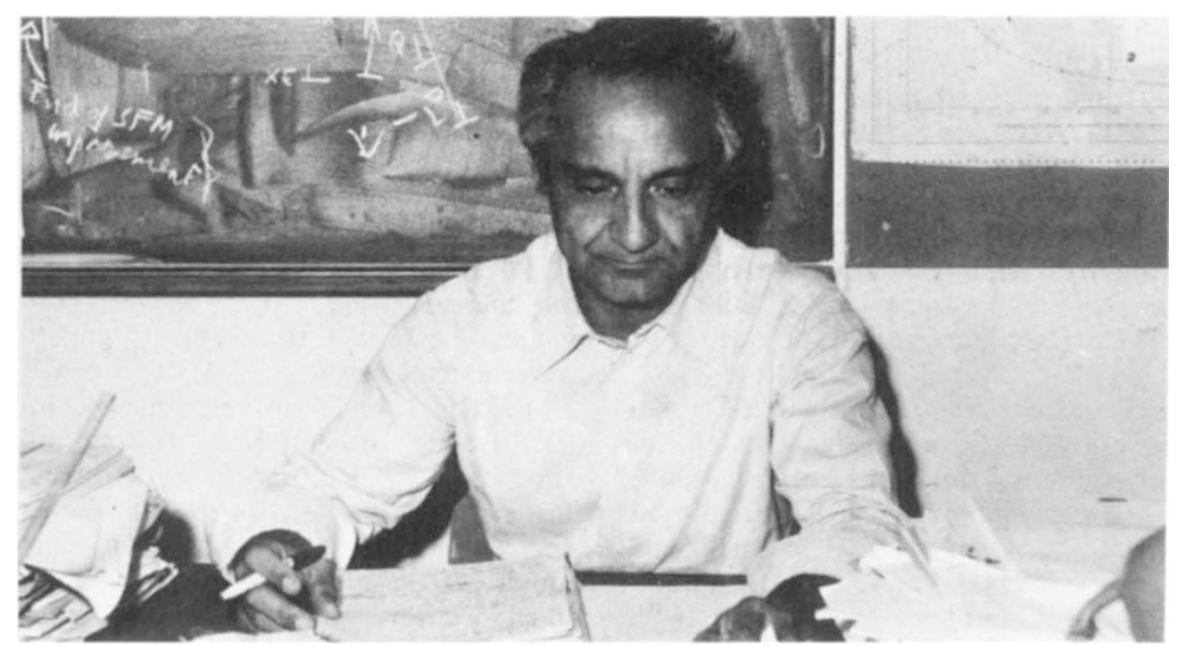

Antonino Zichichi: shy of the camera, but not of politicians

\title{
Sicily's scientific evangelist
}

The European Physical Society, which meets next week in York, has for its president a Sicilian very powerful in Italian science politics: Professor Antonino Zichichi. He spoke recently to Robert Walgate

Antonino Zichichi, 48-year-old son of a Sicilian small businessman, entered university without knowing that physics could be a career. Now he is Professor of Advanced Physics at the University of Bologna. He is also virtually king of Erice, a Sicilian hill-top village where 15 years ago he established a summer school: the Ettore Majorana Centre. (A hundred scientists a week now visit Erice. "I had left Sicily. I felt guilty. I wanted to help.") Zichichi has also helped the Italian National Nuclear Physics Institute (INFN), of which he is president. Earlier this month he succeeded in doubling its budget, committing Italy to three new accelerators: an electron storage ring, a superconducting cyclotron, and an electron linac.

Professor Zichichi is a particle physicist who keeps company with the Italian ministers of science and education. And he is now President of the European Physical Society. He is also, without doubt, a thoroughgoing romantic. $\mathrm{He}$ is romantic about Europe. He is romantic about science. But both romances are tinged with realism: he wants to get things done.

'Culture' is an important word in his vocabulary. By it he means largely disseminated 'pure science'. Technologists, he says, are "culturally 300 years in the past; they should understand quantum electrodynamics". According to Zichichi, scientists create "new culture". "The greatest three things that mankind has created are first language, then logic, and finally science". Language needs very little logic, just enough for internal consistency. Logic, invented 2,000 years ago, "is above language; it is something new." The most rigorous form of logic is mathematics; but you can invent anything in it, such as spaces of 25 dimensions. Science, which began with Galileo, is the logic of nature. "Science tells you if you want to believe something just ask nature. It took 2,000 years to learn that force was proportional to acceleration and not to velocity, because the Greeks only argued". Thus Zichichi believes that science is the highest achievement of mankind.

"But science has no impact in human culture. Human culture is still dominated by language. People can still be influenced by a slogan. Invent a slogan and you will have millions of people following you. A slogan is an insult to human intelligence." Science, says Zichichi, "has produced a lot of science but very little culture." What would be poetry "if poets had kept their work only in a closed drawer, or communicated only among themselves? Or painting if Raphael or Picasso had kept their paintings locked away? Or music if musicians had played only to each other? With science it's the same story. There are immense treasuries of knowledge locked away in our laboratories and our libraries, but scientists have done very little to make them known to everybody."

There is a barrier to cross in that one has to work to understand science; but 300,000 years ago probably very few people had language. Using a language is not easy; but now everyone can speak. Why? Because we hear speech from birth. "My conviction is that the same should happen with science. One day everybody will be scientists-not in the sense that they will make discoveries, but they will understand the discoveries that others are making, which is already a very great step forward".

Professor Zichichi is not unaware of the more temporal benefits of publicity. I was interviewing him at CERN, the European nuclear physics laboratory near Geneva, where he has spent most of his career since 1956. I asked him what he considered his greatest contribution to physics. It was the discovery of the antideuteron-a nucleus consisting of an antiproton and an antineutron-which he made in one night in 1965. "If you forgot the accepted wisdom about invariance principles and just thought classically, you could ask who told us that the antiproton and the antineutron stuck together like the proton and the neutron? And if they did not, the whole route to the antinuclei would have been stopped." Zichichi found the antideuteron. "And we didn't make any press release-nothing." Seven years later another group rediscovered it and published it widely. "At this point I decided to take more care of public impact".

Zichichi began to write for an Italian newspaper — "the greatest", Corriere della Sera, in a column titled 'new culture'. "I don't like to call it science popularisation". After two years he moved to Il Giornale Nuove, to reach a new public, and now he writes articles of up to 1,000 words for the Saturday edition of the Rome newspaper Il Tempo. The latter has a circulation of 300,000 , half that of his first paper, but it is read by "political people, religious people, in Rome". It is therefore more influential; and Zichichi gets more letters about his $I l$ Tempo articles than any others.

If communicating science is one of Zichichi's passions, then so also are people. He sees European Physical Society first in terms of creating friendships; he sees his negotiations with ministers in terms of relationships and conversations, as a matter of convincing individuals face-to-face with his arguments. "I like to leave the administration of INFN to those who can do it better than me. I work on the scientific strategy; I try to convince people at the highest level with good arguments ... Politicians are not stupid people. They are very smart. Too many of my colleagues make the mistake of thinking them stupid."

I asked Zichichi what was the place of a scientist in Italian politics: "Nonexistent."

"But you have a lot of influence."

"I am the exception not the rule." 
"How do you do it?"

"I don't know how I do it. I think it's because they understand me and not the others."

And yet "I don't like to waste my time with politics. I took the presidency of INFN because I thought we were going to have troubles. The first thing I like to do is the research I am interested in. But you cannot spend your life complaining . . . some time ago I was arguing about strategy and so on with my colleagues in INFN, and at a certain point they said well, you cannot refuse to be president if you really want to go on like this. I would have preferred someone else to step in; but $I$ realised $I$ had to accept responsibility for the things I wanted to get done."

"Do you have any other administrative ambitions?"

"No. Zero. Absolutely no." I asked about CERN. "In CERN I do only physics. I've never had any responsibility of any kind. And I'm very glad, for there must be a place where you do only physics." Could CERN be better run? He was not in a position to say. "It is always easy to criticise organisations. I have a positive attitude to anything existing. But what I can tell you is that it is easy for big organisations to slip out of purely scientific control. I am facing this problem at INFN, and that is only onetenth the size of CERN."

"Would you like to be director of CERN?"

"No. Because if you are director of CERN you cannot do any more physics. And I want to do physics. It is all right for a theorist. But if you are an experimenter you must make a choice: either you are director of CERN or you do physics. The games I am in now, I can combine with my research; I would feel without an important part of myself if I had to give up physics."

Erice perhaps weighs equally with research in Zichichi's constitution: "I am in touch by telephone for a few minutes every day".' Why did he start the summer school? For a complex of reasons. "As you know Sicily is a place of great culture. Pretty well every culture but the Chinese has been through Sicily. But it is an underdeveloped part of Europe. I was not living there, and in a certain sense I felt guilty. But what could I do?

"One thing was clear to me. The universities started 1,000 years ago because people wanted to know what was going on at the frontier or knowledge. Books were very slow. So people had to meet. But now universities cannot bring people to the frontier because of the great development of modern knowledge. So the old university spirit has been lost.
"People still want to be brought to the frontier. But who is going to teach you? It may be that at $\mathrm{X}$ you have the greatest world specialist in some discipline and you are lucky; but you miss the other disciplines. So if you can establish a place where people can be brought to the frontier of various fields then they will come. That is the principle of the Ettore Majorana Centre at Erice."

The school is financed by the Sicilian Government (Sicily is independent of Italy but for foreign policy), by INFN, and occasionally by other bodies such as NATO. When it started Zichichi had offers of buildings. "But underdeveloped countries are strewn with cathedrals in the desert. I wanted to be sure this place would work. And Erice beautiful, of human dimensions."

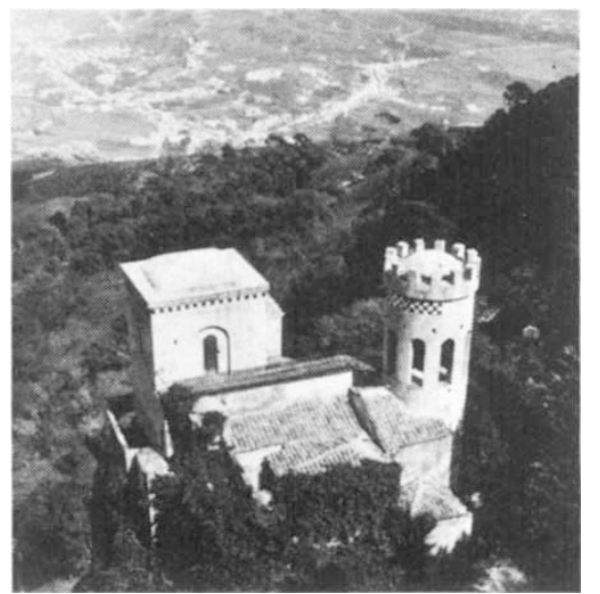

Erice, home of Zichichi's summer school.

Erice is built on a volcanic outcrop of rock, like a natural fortress. On one street you walk on the same stones as the Romans; more recently it was like a Switzerland in Sicily, housing and protecting the richest families. Zichichi's office is the highest part of Erice. "The view from there is absolutely fantastic." The 'summer' school (it lasts from March to December) is the whole economy of the town, and, according to one enthusiastic visitor the few inhabitants (some 100 or so, says Zichichi) bow their heads to Zichichi as he passes.

Zichichi hands out prizes to the best students (in fistfuls of Lire at the end of a course, an ex-student says) "but it is not easy to win a prize. The people who go there are really very brilliant. It is a pleasure to be there."

In March this year Zichichi 'emerged' as President of the European Physical Society, through a similar process to the one that found him president of the INFN: he wanted to do things. Following in the footsteps of his predecessor-and father-in-law-Gilberto Bernadini he has a romantic ideal for the EPS, uniting East and West and forging links among nations: "We should contribute to the cultural unification of Europe."

Zichichi also sees the EPS as a source of "one of the greatest human feelings", friendship. "In the present age when there is an enormous amount of communication and we know what is going on on the other side of the world, we don't know our neighbours two metres away. And it is very important for physicists to talk, to get to know each other."

The EPS should also play a role in the five great physics projects in Europe: a giant proton synchrotron (probably in the USSR); a very large electron-positron colliding beam machine; JET; a large synchrotron radiation laboratory; and a millimetre wave radio interferometer. It should also develop proposals on the physics components of two new teaching institutions at present under discussion in Europe: a European 'science university' and a 'Mediterranean polytechnic' for the underdeveloped regions of the Mediterranean. And it should encourage the exchange of students and professors, perhaps for whole periods of a year's teaching: "imagine the impact of a London professor of quantum mechanics teaching in say Palermo, or Leningrad, for a whole year. Sabbatticals are not enough. We need to interest governments in this."

In the end Zichichi will always come back to his physics. He is an unconventional researcher. He was the first to look at the production of leptons in collisions of hadrons, work which eventually led to a Nobel Prize for Samuel Ting in the discovery of the 'hidden charm' particle J/psi. "Ting mentioned me in his Nobel address as the pioneer. I can be proud and ashamed-proud that I started, and ashamed that I gave up". Also he spent five years searching for a lepton heavier than the muon at Frascati, by looking at electron annihilations producing an electron, a muon, and anything-the same reaction in which Martin Perl at Stanford discovered the 1.7 GeV tau lepton ten years later (the tau was just above the Frascati energy, like the $J / \mathrm{psi}$ itself. There is little doubt that Frascati's energy was very unfortunately chosen.)

Now Zichichi is "trying to break the proton". The concept that the quarks are infinitely bound into the proton "is only at the level of "theoretical feeling'" says Zichichi. "There is no proved theorem that says they should stay in there." Zichichi, while admiring elegant theory, has no truck with theoretical speculation, any more than he has time for reading the fantasies of novelists. In the matter of the proton he wants to ask Nature her logic. He has, as he puts it, "a very realistic view". 